\title{
Solitary wave solutions to two nonlinear evolution equations via the modified simple equation method
}

\author{
Jesmin Akter ${ }^{1}$ and M. Ali Akbar ${ }^{2}$ \\ ${ }^{1}$ Department of Natural Science, Varendra University, Bangladesh \\ ${ }^{2}$ Department of Applied Mathematics, University of Rajshahi, Bangladesh
}

Received: 15 February 2016, Accepted: 21 March 2016

Published online: 8 October 2016.

\begin{abstract}
In this article, we investigate two essential nonlinear evolution equations namely modified dispersive water wave equations and the Whitham-Broer-Kaup model for dispersive long waves in the shallow water small-amplitude regime by using the modified simple equation (MSE) method. The obtained solutions with parameters expose that the method is incredibly prominent and effective mathematical tool for solving nonlinear evolution equations (NLEEs) in mathematical physics, applied mathematics and engineering. When the parameters have taken special values the solitary wave solutions are attained from the exact solutions. In addition, this procedure reduces the size of calculations.
\end{abstract}

Keywords: Modified simple equation method, nonlinear evolution equations, solitary wave solutions, modified dispersive water wave equations, Whitham-Broer-Kaup model for dispersive long waves in the shallow water small-amplitude regime.

\section{Introduction}

Nonlinear evolution equations (NLEEs) have a vast area for playing a big role and it is very essential for the calculating mathematical equation of modern science. The maximum complex phenomena come out in a extensive variety of scientific applications, such as, the fluid dynamics, chemical kinematics, plasma physics, nuclear physics, applied mathematics, biology, solid state physics, optical fibers, and so on are modeled by nonlinear evolution equation. Traveling wave solution is a fundamental problem for this model to seek their analytical solution. In soliton theory the traveling wave solutions of NLEEs have provided a significant and imperative role, as they provide a great deal of information about the physical models they express. Numerous mathematician and physical scientists have made to obtain the exact solutions and solitary wave solutions of these NLEEs which rotate into an essential task in the study of nonlinear physical phenomena. It is well mentioned that there is no unique method to solve the all kind of NLEEs and in this regards a lot of new techniques have been successfully developed by diverse group of scientists such as, the homotopy perturbation method [1-3], the Jacobi elliptic function method [4], the truncated Painleve expansion method [5], He's semi-inverse variational principle [6-7], the variational method [8-11], the Backlund transformation [12], the Miura transformation [13], the F-expansion method [14], the generalized Riccati equation [15], the homogenous balance method [16-17], the trial function method [18], the $\exp (-\varphi(\xi))$-expansion method [19-21], the tanh-function method [22-24], the inverse scattering method [25], the sine-cosine method [26-27], the Exp-function method [28-30], the asymptotic method [31], the Hirota's bilinear transformation method [32-33], the auxiliary equation method [34], the non-perturbative method [35], method of integrability [36], the soliton perturbation theory [37-39], the $\left(G^{\prime} / G\right)$-expansion method [40-47], etc.

The main purpose of this article is to make use of the MSE method to extract new exact traveling wave solutions and 
then solitary wave solutions to the modified dispersive water wave equations and Whitham-Broer-Kaup model for dispersive long waves in the shallow water small-amplitude regime and reveal the progress and straightforwardness of this method. Moreover the MSE method has not been applied to the above pointed out equation in the earlier literature. Gozukizil and Aydemir [48] studied modified dispersive water wave equations by using $\left(G^{\prime} / G\right)$-expansion method, Alquran and Qawasmeh [49] investigated Whitham-Broer-Kaup model via $\left(G^{\prime} / G\right)$-expansion method.

The article is prepared as follows: In section 2, the MSE method has been discussed. In section 3, the method is applied into two nonlinear evolution equations mentioned above. In section 4, physical explanations of the obtained solutions have been discussed and in section 5, we have drawn our conclusions.

\section{Outline of the modified simple equation method}

In this section we describe the modified simple equation method for finding exact traveling wave solutions of nonlinear evolution equations. Let us consider the nonlinear partial differential equation for $u(x, t)$ is in the form

$$
Q\left(u, u_{t}, u_{x}, u_{t t}, u_{x t}, u_{x x}, \ldots .\right)=0
$$

Here $Q$ is a polynomial in $u(x, t)$ and its various partial derivatives, in which the highest order derivatives and nonlinear terms are involved. In order to solve $u(x, t)$ explicitly we have to carry out the following subsequential four steps:

Step 1. Combining the independent variables $x$ and $t$ into a compound variable $\xi$, we consider that

$$
u(x, t)=u(\xi), \xi=x-W t
$$

where $W$ is the speed of the travelling wave. The wave variable (2) permits us to convert Eq. (1) into an ordinary differential equation (ODE) for $u=u(\xi)$ :

$$
R\left(u, u^{\prime}, u^{\prime \prime}, u^{\prime \prime \prime}, \ldots . .\right)=0
$$

where $R$ is a polynomial in $u(\xi)$ and its derivatives wherein prime indicates the derivative with respect to $\xi$.

Step 2. Suppose the Eq. (3) has the formal solution

$$
u(\xi)=B_{0}+\sum_{k=0}^{N} B_{k}\left[\frac{\psi^{\prime}(\xi)}{\psi(\xi)}\right]^{k}
$$

where $B_{k}(k=1,2,3, \cdots)$ are arbitrary constants to be calculated, such that $B_{N} \neq 0$, and $\psi(\xi)$ is an unknown function to be determined subsequently. The solution which has prepared by the $\left(G^{\prime} / G\right)$-expansion method, Exp-function method, Jacobi elliptic function method, etc., presented in terms of some pre-settled functions, but in the MSE method, $\psi$ is not previously known or not a solution of any known equation. For that reason, it is impossible to speculate from previous what kind of solutions one may get through this method. This is the distinction and exquisiteness of this method.

Step 3. The positive integer $N$ come out in Eq. (4) can be determined by taking into consideration the balance between the highest order derivatives and highest order nonlinear terms occurring in Eq. (3).

Step 4. Calculating the necessary derivatives $u^{\prime}, u^{\prime \prime}, u^{\prime \prime \prime} \cdots$ of a function $u(\xi)$ and substituting Eq. (4) into (3), we explicate the function $\psi(\xi)$. As a result of this replacement we get a polynomial in $\left(\psi^{\prime}(\xi) / \psi(\xi)\right)$ and its derivatives. Equating all the coefficients with like power of this polynomial to zero, we obtain an over determined set of equations 
which can be solved to find $B_{k}(k=0,1,2,3, \cdots)$ and $\psi(\xi)$. This accomplished the determination of the solution of Eq. (1).

\section{Applications of the method}

In this section, two examples have been utilized to demonstrate the distinction and inevitability of the MSE method to construct exact solutions and then solitary wave solutions of nonlinear partial differential equations.

\subsection{The modified dispersive water wave equations}

In this sub-section, we will bring to bear the MSE method for finding exact solitary wave solutions to the modified dispersive water wave equations. Let us consider the modified dispersive water wave equations (MDWWE) are given by $u_{t}=-\frac{1}{4} v_{x x}+\frac{1}{2}\left(u v_{x}+v u_{x}\right)$

$$
v_{t}=-u_{x x}-2 u u_{x}+\frac{3}{2} v v_{x}
$$

By making use of the traveling wave variable $\xi=x-W t$ into Eq. (5) we obtain the following ODEs:

$$
\begin{gathered}
-W u^{\prime}=-\frac{1}{4} v^{\prime \prime}+\frac{1}{2}\left(u v^{\prime}+v u^{\prime}\right), \\
-W v^{\prime}=-u^{\prime \prime}-2 u u^{\prime}+\frac{3}{2} v v^{\prime} .
\end{gathered}
$$

Integrating Eq. (6) with respect to $\xi$ once and choosing the integration constant as zero, we obtain

$$
\begin{gathered}
-W u=-\frac{1}{4} v^{\prime}+\frac{1}{2} u v, \\
-W v=-u^{\prime}-u^{2}+\frac{3}{4} v^{2} .
\end{gathered}
$$

Now balancing the highest-order derivatives and the nonlinear terms occurring in Eq. (7), we obtain $M=1$ and $N=1$. Consequently, the solution of Eq. (5) takes the following form

$$
\begin{aligned}
& u=A_{0}+A_{1}\left(\frac{\psi^{\prime}}{\psi}\right), \\
& v=B_{0}+B_{1}\left(\frac{\psi^{\prime}}{\psi}\right),
\end{aligned}
$$

wherein $A_{0}, A_{1}, B_{0}$ and $B_{1}$ are constants, such that $\left(A_{1}, B_{1} \neq 0\right)$, and $\psi(\xi)$ is an undefined function to be determined later. The essential computations for the equation (7) are as follows:

$$
\begin{aligned}
& u^{\prime}=A_{1}\left[\frac{\psi^{\prime \prime}}{\psi}-\left(\frac{\psi^{\prime}}{\psi}\right)^{2}\right] . \\
& v^{\prime}=B_{1}\left[\frac{\psi^{\prime \prime}}{\psi}-\left(\frac{\psi^{\prime}}{\psi}\right)^{2}\right] .
\end{aligned}
$$




$$
\begin{aligned}
& u^{2}=A_{0}^{2}+2 A_{0} A_{1} \frac{\psi^{\prime}}{\psi}+A_{1}^{2}\left(\frac{\psi^{\prime}}{\psi}\right)^{2} . \\
& v^{2}=B_{0}^{2}+2 B_{0} B_{1} \frac{\psi^{\prime}}{\psi}+B_{1}^{2}\left(\frac{\psi^{\prime}}{\psi}\right)^{2} .
\end{aligned}
$$

Substituting the values of $u^{\prime}, v^{\prime}, u^{2}$ and $v^{2}$ into Eq. (7) and then equating the coefficients of $\psi^{0}, \psi^{-1}, \psi^{-2}$ to zero, yields

$$
\begin{gathered}
-W A_{0}-\frac{1}{2} A_{0} B_{0}=0 . \\
-W A_{1} \psi^{\prime}+\frac{1}{4} B_{1} \psi^{\prime \prime}-\frac{1}{2} A_{0} B_{1} \psi^{\prime}-\frac{1}{2} A_{1} B_{0} \psi^{\prime}=0 \\
-\frac{1}{4} B_{1} \psi^{\prime 2}-\frac{1}{2} A_{1} B_{1} \psi^{\prime 2}=0 . \\
-W B_{0}+A_{0}^{2}-\frac{3}{4} B_{0}^{2}=0 . \\
-W B_{1} \psi^{\prime}+A_{1} \psi^{\prime \prime}+2 A_{0} A_{1} \psi^{\prime}-\frac{3}{2} B_{1} B_{0} \psi^{\prime}=0 . \\
-A_{1} \psi^{\prime 2}+A_{1}^{2} \psi^{\prime 2}-\frac{3}{4} B_{1}^{2} \psi^{\prime 2}=0 .
\end{gathered}
$$

From Eqs. (13), (15), (16) and (18) we obtain

$$
B_{0}=-2 W, A_{1}=-\frac{1}{2}, A_{0}= \pm W \text { and } B_{1}= \pm 1
$$

Solving Eqs. (14) and (16), then substituting (19) into Eq. (8) we obtain

$$
u= \pm W-\frac{1}{2}\left(\frac{C_{1} e^{L \xi}}{C_{2}+\frac{C_{1}}{L} e^{L \xi}}\right)
$$

and

$$
v=-2 W \pm\left(\frac{C_{1} e^{M \xi}}{C_{2}+\frac{C_{1}}{M} e^{M \xi}}\right)
$$

where

$$
L=\frac{4}{B_{1}}\left(w A_{1}+\frac{1}{2} A_{0} B_{1}+\frac{1}{2} A_{1} B_{0}\right), M=\frac{1}{A_{1}}\left(w B_{1}-2 A_{0} A_{1}+\frac{3}{2} B_{1} B_{0}\right),
$$

$C_{1}$ and $C_{2}$ are constants of integration. Since $C_{1}$ and $C_{2}$ are constants of integration, we might explicitly pick their values. If we choose $C_{1}=L$ and $C_{2}=1$ then the solutions (20) and (21) reduce to,

$$
u(x, t)= \pm W-\frac{1}{4} L\left[1+\tanh \left(\frac{1}{2} L(x-W t)\right)\right]
$$

and

$$
v(x, t)=-2 W+\frac{1}{2} M\left[1+\tanh \left(\frac{1}{2} M(x-W t)\right)\right]
$$


Otherwise, if we choose $C_{1}=-L$ and $C_{2}=1$ then the solutions (20) and (21) reduce to,

$$
u(x, t)= \pm W-\frac{1}{4} L\left[1+\operatorname{coth}\left(\frac{1}{2} L(x-W t)\right)\right]
$$

and

$$
v(x, t)=-2 W+\frac{1}{2} M\left[1+\operatorname{coth}\left(\frac{1}{2} M(x-W t)\right)\right] .
$$

Using hyperbolic function identities Eqs. (23), (24), (25) and (26) can respectively be rewritten as

$$
u(x, t)= \pm W-\frac{1}{4} L\left[1-i \tan \left(\frac{i}{2} L(x-W t)\right)\right],
$$

and

$$
\begin{gathered}
v(x, t)=-2 W+\frac{1}{2} M\left[1-i \tan \left(\frac{i}{2} M(x-W t)\right)\right] . \\
u(x, t)= \pm W-\frac{1}{4} L\left[1+i \cot \left(\frac{i}{2} L(x-W t)\right)\right],
\end{gathered}
$$

and

$$
v(x, t)=-2 W+\frac{1}{2} M\left[1+i \cot \left(\frac{i}{2} M(x-W t)\right)\right] .
$$

Remark. Solutions (20)-(30) have been verified by substituting them back into the original equation and found correct.

\subsection{Whitham-Broer-Kaup model for dispersive long waves in the shallow water small-amplitude regime}

Let us consider the Whitham-Broer-Kaup model for dispersive long waves in the shallow water small-amplitude regime is in the following form

$$
\begin{gathered}
u_{t}+u u_{x}+v_{x}+\beta u_{x x}=0, \\
v_{t}+(u v)_{x}-\beta v_{x x}+\alpha u_{x x x}=0 .
\end{gathered}
$$

This is completely integrable model describes the dispersive long wave in shallow water, where $u(x, t)$ is the horizontal velocity, $v(x, t)$ is the height which diverges from the equilibrium position of liquid. $\alpha$ and $\beta$ are constants that characterize different diffusion powers. It is a superior model to describe the dispersive long wave. If $\beta=0$ and $\alpha=1$ then this system represent variant Boussinesq equation.

The traveling wave transformation $u(\xi)=u(x, t), \xi=x-W t$ reduces the Eq. (31) into the following ODEs:

$$
\begin{gathered}
-W u^{\prime}+u u^{\prime}+v^{\prime}+\beta u^{\prime \prime}=0, \\
-W v^{\prime}+(u v)^{\prime}-\beta v^{\prime \prime}+\alpha u^{\prime \prime \prime}=0 .
\end{gathered}
$$


By integrating Eq. (32) once with respect to $\xi$ and neglecting the constant of integration, we obtain

$$
\begin{aligned}
& -W u+\frac{u^{2}}{2}+v+\beta u^{\prime}=0, \\
& -W v+u v-\beta v^{\prime}+\alpha u^{\prime \prime}=0 .
\end{aligned}
$$

From Eq. (33) we have

$$
v=W u-\frac{u^{2}}{2}-\beta u^{\prime}
$$

Substituting Eq. (35) into (34) we obtain

$$
-W^{2} u+\frac{3}{2} W u^{2}-\frac{1}{2} u^{3}+\left(\beta^{2}+\alpha\right) u^{\prime \prime}=0
$$

Balancing the highest-order derivative $u^{\prime \prime}$ and the nonlinear term $u^{3}$, we obtain $N=1$. Consequently we get the formal solution

$$
u(\xi)=A_{0}+A_{1}\left(\frac{\psi^{\prime}}{\psi}\right),
$$

where $A_{0}$ and $A_{1}$ are arbitrary constants such that $A_{1} \neq 0$, and $\psi(\xi)$ is an unknown function to be determined afterward. It is easy to make out,

$$
\begin{gathered}
u^{\prime \prime}=A_{1} \frac{\psi^{\prime \prime \prime}}{\psi}-3 A_{1} \frac{\psi^{\prime \prime} \psi^{\prime}}{\psi^{2}}+2 A_{1}\left(\frac{\psi^{\prime}}{\psi}\right)^{3} . \\
u^{2}=A_{0}^{2}+2 A_{0} A_{1} \frac{\psi^{\prime}}{\psi}+A_{1}^{2}\left(\frac{\psi^{\prime}}{\psi}\right)^{2} . \\
u^{3}=A_{0}^{2}+3 A_{0}^{2} A_{1} \frac{\psi^{\prime}}{\psi}+3 A_{0} A_{1}^{2}\left(\frac{\psi^{\prime}}{\psi}\right)^{2}+A_{1}^{3}\left(\frac{\psi^{\prime}}{\psi}\right)^{3} .
\end{gathered}
$$

Substituting Eqs. (38), (39) and (40) into Eq. (36) yields a polynomial in $\frac{1}{\psi^{j}},(j=0,1,2, \cdots)$ and equating the coefficients of $\psi^{0}, \psi^{-1}, \psi^{-2}, \psi^{-3}$ to zero, yields

$$
\begin{gathered}
-W^{2} A_{0}+\frac{3}{2} W A_{0}^{2}-\frac{1}{2} A_{0}^{3}=0 \\
-W^{2} A_{1} \psi^{\prime}+3 W A_{0} A_{1} \psi^{\prime}-\frac{3}{2} A_{1} A_{0}^{2} \psi^{\prime}+\left(\beta^{2}+\alpha\right) A_{1} \psi^{\prime \prime \prime}=0 \\
\frac{3}{2} W A_{1}^{2} \psi^{\prime 2}-\frac{3}{2} A_{0} A_{1}^{2} \psi^{\prime 2}-\left(\beta^{2}+\alpha\right) 3 A_{1} \psi^{\prime} \psi^{\prime \prime}=0 \\
-\frac{1}{2} A_{1}^{3} \psi^{\prime 3}+\left(\beta^{2}+\alpha\right) 2 A_{1} \psi^{\prime 3}=0
\end{gathered}
$$

Solving Eq. (41), we obtain

$$
A_{0}=0, W, 2 W
$$

Since $A_{1} \neq 0$, from Eq. (44), we obtain

$$
A_{1}= \pm 2 \sqrt{\beta^{2}+\alpha}
$$

Case 1. When $A_{0}=W$, Eq. (42) and Eq. (43) yields a trivial solution. So this case is rejected. 
Case 2. When $A_{0}=0$, Eq. (42) and Eq. (43) yields

$$
\frac{\psi^{\prime \prime \prime}}{\psi^{\prime \prime}}=P Q
$$

where $P=\frac{\beta^{2}+\alpha}{\frac{1}{2} W A_{1}}$ and $Q=\frac{W^{2}}{\beta^{2}+\alpha}$. Integrating Eq. (47) twice with zero constant of integration for the first time and $W \neq 1$, yields

$$
\begin{gathered}
\psi^{\prime}=\frac{C_{1}}{P Q} e^{P Q \xi}, \\
\psi=C_{2}+\frac{C_{1}}{P^{2} Q^{2}} e^{P Q \xi},
\end{gathered}
$$

where $C_{1}$ and $C_{2}$ are arbitrary constants.

Substituting the values of $A_{1}$, Eq. (48) and Eq. (49) into Eq. (37), we obtain following the exact solution to the WhithamBroer-Kaup model

$$
u(\xi)= \pm 2 \sqrt{\beta^{2}+\alpha}\left(\frac{C_{1} P Q e^{P Q \xi}}{C_{2} P^{2} Q^{2}+C_{1} e^{P Q \xi}}\right),
$$

where $\xi=x-W t$. Since $C_{1}$ and $C_{2}$ are constants of integration, we might explicitly pick their values. If we choose $C_{1}=1$ and $C_{2}=\frac{\beta^{2}+\alpha}{W}$ then the solution (50) turns into,

$$
u_{1,2}(x, t)= \pm W\left(1+\tanh \left(\frac{1}{2} \frac{W}{\sqrt{\beta^{2}+\alpha}}(x-W t)\right)\right)
$$

On the other hand, if we choose $C_{1}=-1$ and $C_{2}=\frac{\beta^{2}+\alpha}{W}$ then the solution (50) turns into,

$$
u_{3,4}(x, t)= \pm W\left(1+\operatorname{coth}\left(\frac{1}{2} \frac{W}{\sqrt{\beta^{2}+\alpha}}(x-W t)\right)\right)
$$

Now applying (51) and (52) into Eq. (35) we obtain

$$
\begin{aligned}
& v_{1,2}(x, t)=\frac{W^{2}}{2}\left(1 \mp \frac{\beta}{\sqrt{\beta^{2}+\alpha}}\right)\left(1-\tanh ^{2}\left(\frac{1}{2} \frac{W}{\sqrt{\beta^{2}+\alpha}}(x-W t)\right)\right) . \\
& v_{3,4}(x, t)=\frac{W^{2}}{2}\left(1 \mp \frac{\beta}{\sqrt{\beta^{2}+\alpha}}\right)\left(1-\operatorname{coth}^{2}\left(\frac{1}{2} \frac{W}{\sqrt{\beta^{2}+\alpha}}(x-W t)\right)\right) .
\end{aligned}
$$

Using hyperbolic function identities Eqs. (51), (52), (53) and (54) can respectively be rewritten as

$$
\begin{gathered}
u_{5,61}(x, t)= \pm W\left(1-i \tan \left(\frac{i}{2} \frac{W}{\sqrt{\beta^{2}+\alpha}}(x-W t)\right)\right) . \\
u_{7,8}(x, t)= \pm W\left(1+i \operatorname{coth}\left(\frac{i}{2} \frac{W}{\sqrt{\beta^{2}+\alpha}}(x-W t)\right)\right) . \\
v_{5,6}(x, t)=\frac{W^{2}}{2}\left(1 \mp \frac{\beta}{\sqrt{\beta^{2}+\alpha}}\right)\left(1+\tan ^{2}\left(\frac{1}{2} \frac{W}{\sqrt{\beta^{2}+\alpha}}(x+W t)\right)\right) .
\end{gathered}
$$




$$
v_{7,8}(x, t)=\frac{W^{2}}{2}\left(1 \mp \frac{\beta}{\sqrt{\beta^{2}+\alpha}}\right)\left(1-\cot ^{2}\left(\frac{1}{2} \frac{W}{\sqrt{\beta^{2}+\alpha}}(x+W t)\right)\right) .
$$

Remark. Solutions (50)-(58) have been verified by replacing them back into the original equation and found correct.

Case 3. When $A_{0}=2 W$, we obtain same result included in Eq. (51) - Eq. (58).

\section{Physical explanations}

In this segment, we discuss the physical explanations of the resolute exact solutions and then solitary solutions of the modified dispersive water wave equations and Whitham-Broer-Kaup model for dispersive long waves in the shallow water small-amplitude regime.

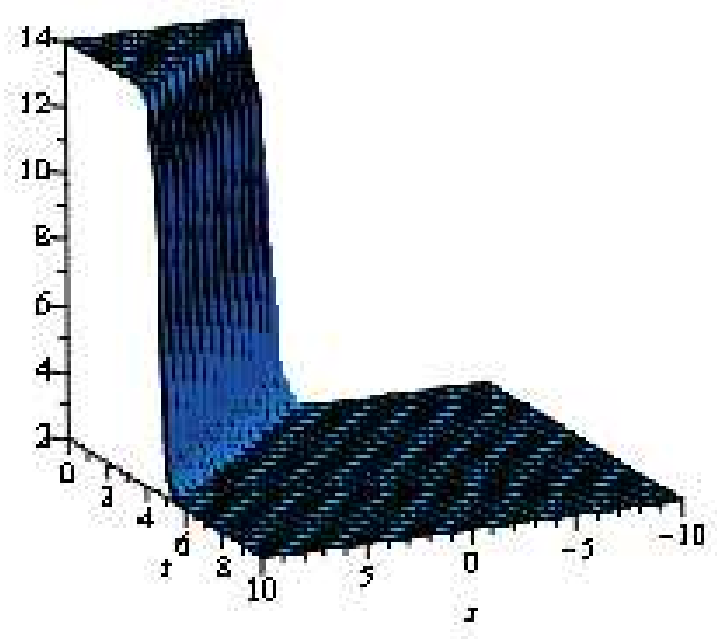

Fig.1 Shape of solution (20) with $W=2, L=4 C_{1}=1, C_{2}=2$ within the interval $(-10 \leq x \leq 10)$ and $(0 \leq t \leq 10)$. 


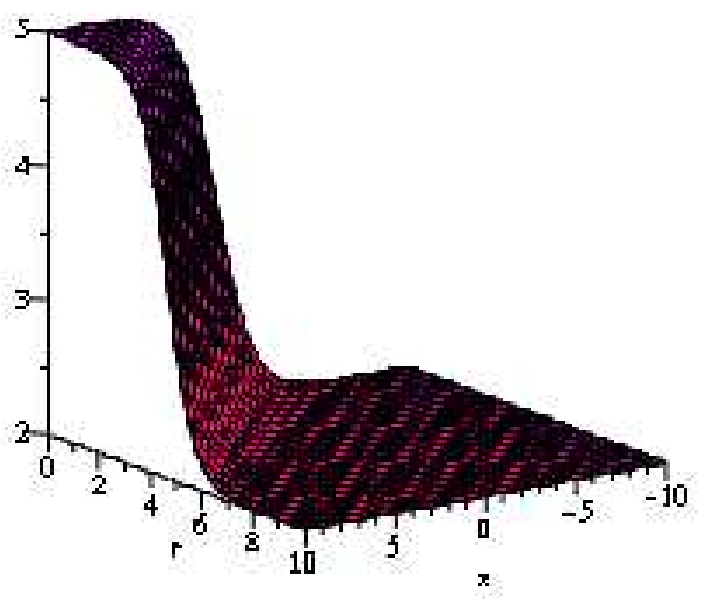

Fig.2 Shape of solution (21) with $W=2, M=1, C_{1}=1, C_{2}=2$ within the interval $(-10 \leq x \leq 10)$ and $(0 \leq t \leq 10)$.

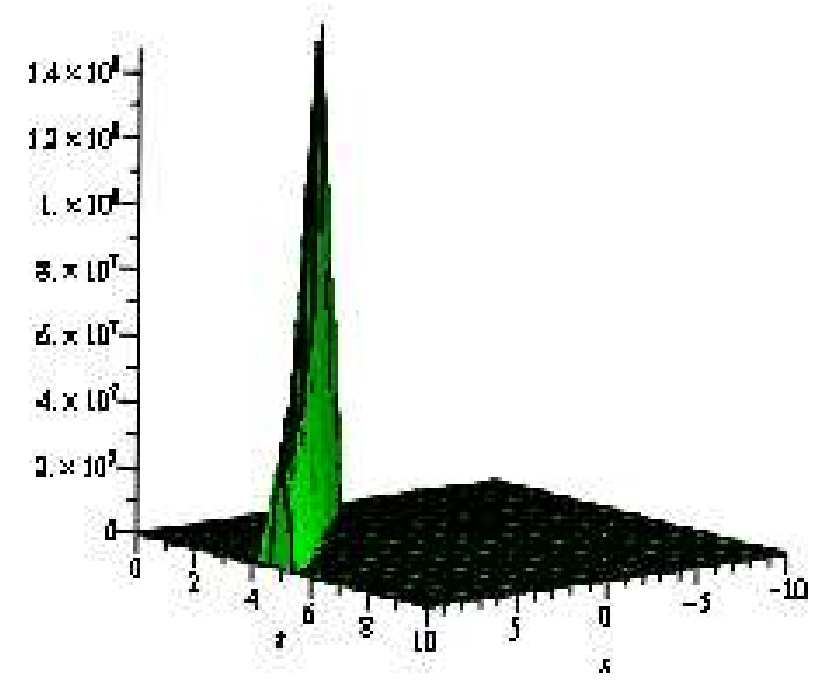

Fig.3 Shape of Eq. (25) with $W=2, L=3$ within the interval $(-10 \leq x \leq 10)$ and $(0 \leq t \leq 10)$. 


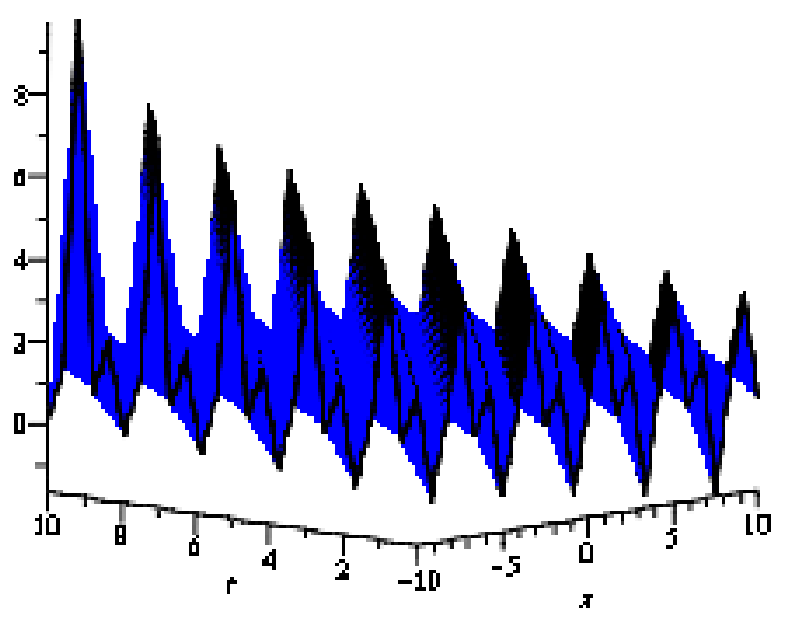

Fig.4 Shape of Eq. (27) with $W=2, L=3$ within the interval $(-10 \leq x \leq 10)$ and $(0 \leq t \leq 10)$.

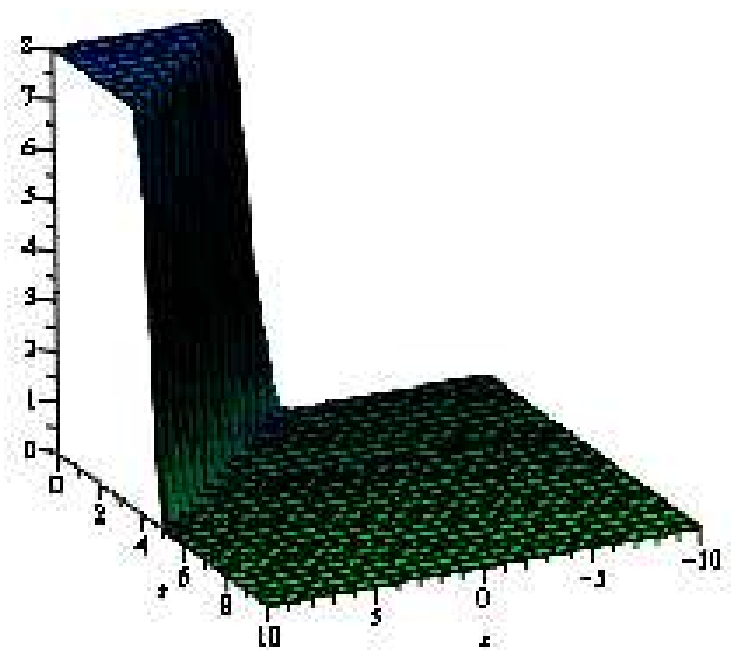

Fig.5 Shape of Eq. (50) with $W=2, P=1, Q=4 C_{1}=1, C_{2}=3$ within the interval $(-10 \leq x \leq 10)$ and $(0 \leq t \leq 10)$. 


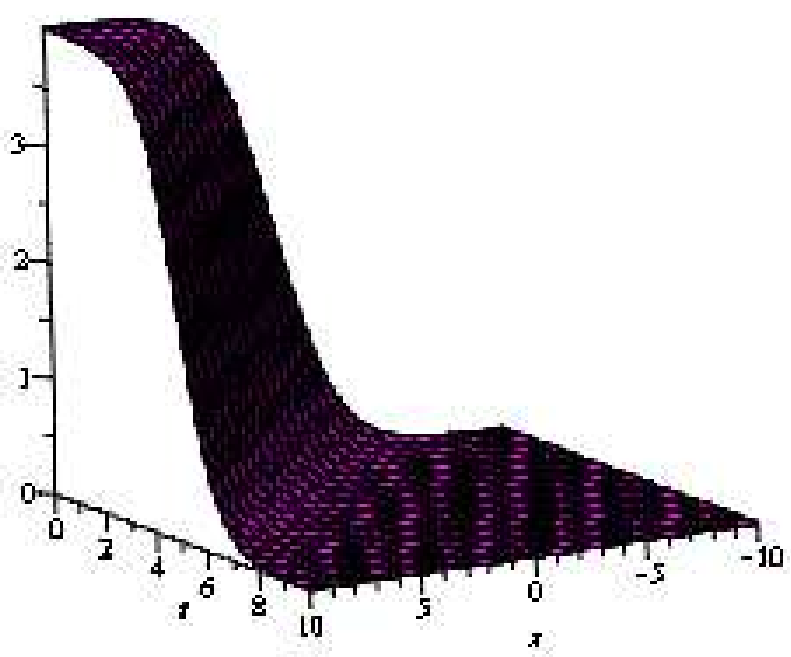

Fig.6 Shape of Eq. (51) with $W=2, \beta=2, \alpha=3$ within the interval $(-10 \leq x \leq 10)$ and $(0 \leq t \leq 10)$.

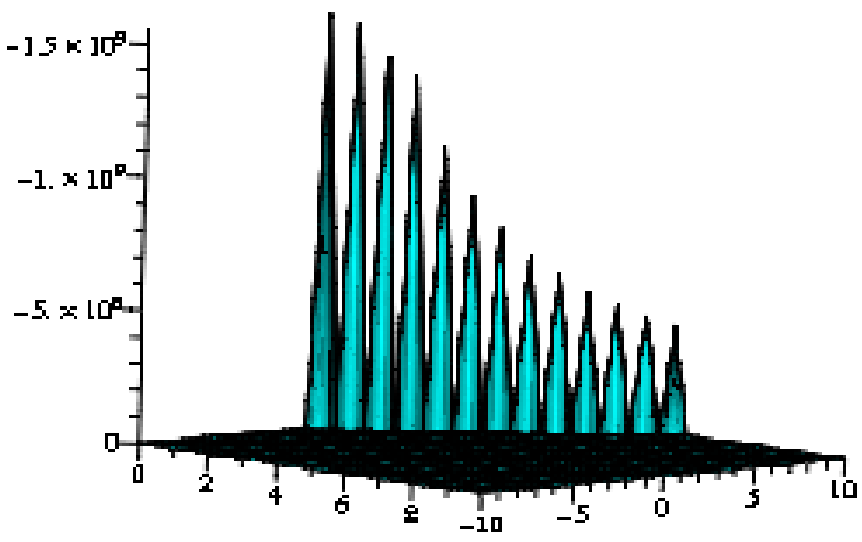

Fig.7 Shape of Eq. (52) with $W=2, \beta=2, \alpha=3$ within the interval $(-10 \leq x \leq 10)$ and $(0 \leq t \leq 10)$. 


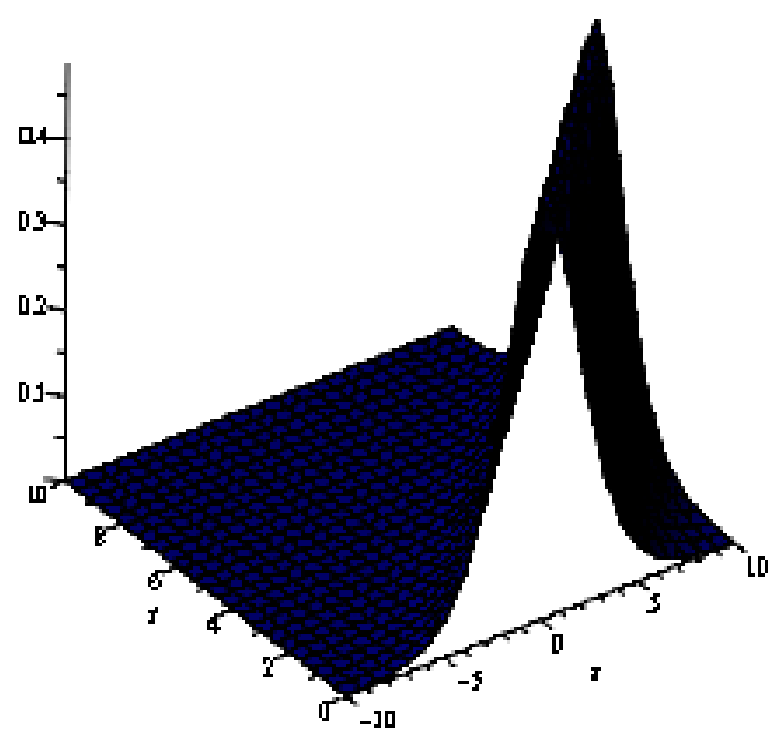

Fig.8 Shape of Eq. (53) with $W=2, \beta=2, \alpha=3$ within the interval $(-10 \leq x \leq 10)$ and $(0 \leq t \leq 10)$.

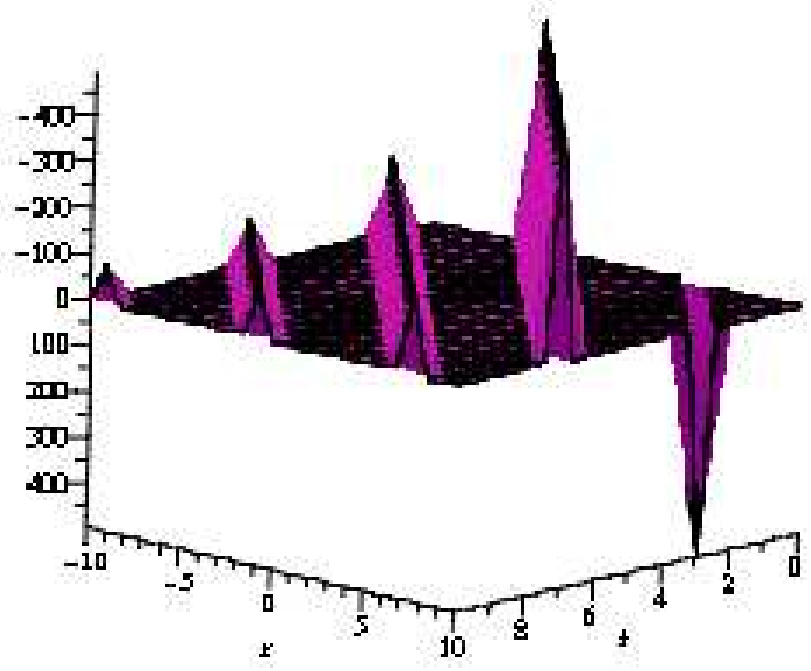

Fig.9 Shape of Eq. (55) with $W=2, \beta=2, \alpha=3$ within the interval $(-10 \leq x \leq 10)$ and $(0 \leq t \leq 10)$. 


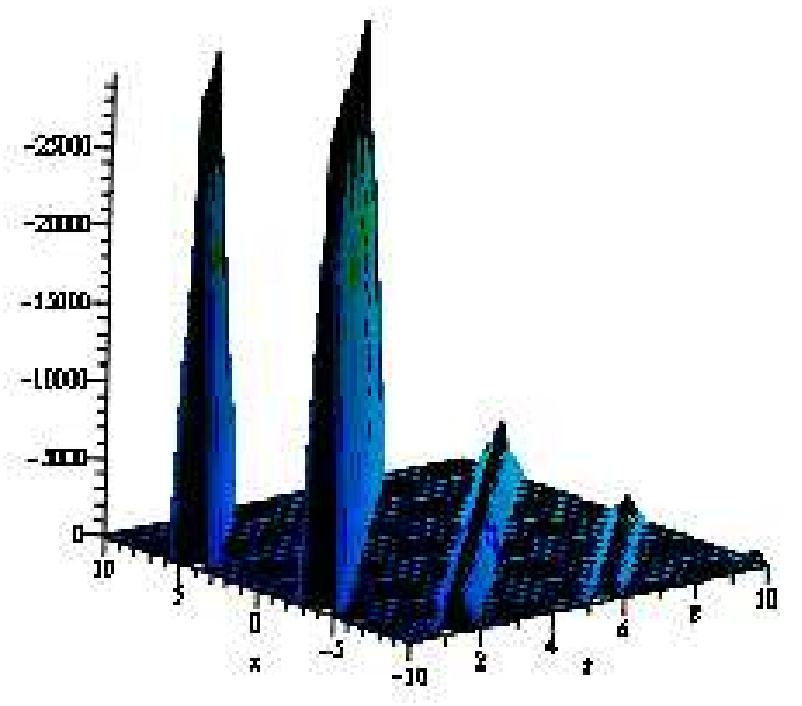

Fig.10 Shape of Eq. (57) with $W=2, \beta=2, \alpha=3$ within the interval $(-10 \leq x \leq 10)$ and $(0 \leq t \leq 10)$.

The solution Eqs. (20), (21), (23), (24) are kink type wave solution. It rise or decent from one asymptotic state $\xi \rightarrow-\infty$ to another asymptotic state $\xi \rightarrow \infty$. Fig.1 shows the shape of solution Eq. (20) which is kink type wave solution with wave speed $W=2, L=4, C_{1}=1, C_{2}=2$ within the interval $-10 \leq x \leq 10$ and $0 \leq t \leq 10$. Fig.2 shows the shape of solution Eq. (21) which is also kink type wave solution with wave speed $W=2, M=1, C_{1}=1, C_{2}=2$ within the interval $-10 \leq x \leq 10$ and $0 \leq t \leq 10$. Fig.3 shows the shape of solution Eq. (25) which is singular kink type solution with wave speed $W=2, L=3$ within the interval $-10 \leq x \leq 10$ and $0 \leq t \leq 10$. Fig. 4 shows the shape of solution Eq. (27) which is exact periodic traveling wave solution with speed $W=2, L=3$ within the interval $-10 \leq x \leq 10$ and $0 \leq t \leq 10$. The solution Eqs. (23), (24), are also kink type wave solutions which are similar to Fig.1 and Fig.2. The solution Eq. (28) is exact periodic traveling wave solution which is similar to Fig.4 and the solution Eqs. (26), (29), (30) are singular kink type wave solutions which are similar to Fig.3, so for simplicity we ignored these figures.

The solution Eqs. (50), (51) of Whitham-Broer-Kaup model are kink type wave solution. It rise or decent from one asymptotic state $\xi \rightarrow-\infty$ to another asymptotic state $\xi \rightarrow \infty$. Fig.5 shows the shape of solution Eq. (50) which is kink type wave solution with wave speed $W=2, P=1, Q=4 C_{1}=1, C_{2}=3$ within the interval $(-10 \leq x \leq 10)$ and $(0 \leq t \leq 10)$. Fig.6 shows the shape of solution Eq. (51) which is kink type wave solution with wave speed $W=2, \beta=2$, $\alpha=3$ within the interval $(-10 \leq x \leq 10)$ and $(0 \leq t \leq 10)$. Fig.7 shows the shape of Eq. (52) which is singular kink type solution with speed $W=2, \beta=2, \alpha=3$ within the interval $(-10 \leq x \leq 10)$ and $(0 \leq t \leq 10)$. Fig.8 shows the shape of Eq. (53) which is exact singular kink type solution with $W=2, \beta=2, \alpha=3$ within the interval $(-10 \leq x \leq 10)$ and $(0 \leq t \leq 10)$. Fig.9 and Fig.10 show the shape of Eq. (55) and (57) respectively which are exact periodic traveling wave solutions with $W=2, \beta=2, \alpha=3$ within the interval $(-10 \leq x \leq 10)$ and $(0 \leq t \leq 10)$. The solution Eqs. (54), (56), (58) are singular kink type wave solutions which are similar to Fig.7, For convenience these figures are omitted.

\section{Conclusions}

In this paper, we implemented successfully the modified simple equation (MSE) method to solve for the coupled modified dispersive water wave and the Whitham-Broer-Kaup model for dispersive long waves in the shallow water small-amplitude regime. The obtained solutions without using any computation system such as Maple, Mathematica etc 
have many prospective applications in nonlinear science. It is significant to show that the modified simple equation (MSE) method is very simple, straightforward and concise. Comparing this method with other methods, like the F-function method, the homotopy perturbation method, the asymptotic method, the non-perturbative method etc, we might conclude that MSE method is more and much simpler than others. This powerful method can also be used to solve many other nonlinear evolution equations which frequently arise in various scientific real time applications. The reliability of the method and the reduction in the size of computational domain give this method a wider applicability.

\section{References}

[1] S.T. Mohyud-Din, A. Yildirim and S. Sariaydin, Numerical soliton solutions of the improved Boussinesq equation, Int. J. Numer. Methods Heat Fluid Flow, 21(7) (2011) 822-827.

[2] S.T. Mohyud-Din, A. Yildirim and G. Demirli, Analytical solution of wave system in $R^{n}$ with coupling controllers, Int. J. Numer. Methods Heat Fluid Flow, 21(2) (2011) 198-205.

[3] S.T. Mohyud-Din, A. Yildirim and S. Sariaydin, Numerical soliton solution of the Kaup-Kupershmidt equation, Int. J. Numer. Methods Heat Fluid Flow, 21(3) (2011) 272-281.

[4] A.T. Ali, New generalized Jacobi elliptic function rational expansion method, J. Comput. Appl. Math., 235 (2011) $4117-4127$.

[5] J.Weiss , M.Tabor and G.Carnevale, The Painleve property for partial differential equations, J. Math. Phys., 24 (1983) 522.

[6] R. Sassaman, A. Heidari and A. Biswas, Topological and non-topological solitons of nonlinear Klein-Gordon equations by He's semi-inverse variational principle, J. Frank. Ins., 347 (2010) 1148-1157.

[7] R. Sassaman and A. Biswas, Soliton solution of the generalized Klein-Gordon equation by semi-inverse variational principle, Math. Eng. Sci. and Aeros., 2 (2011) 99-104.

[8] J.H. He, Variational iteration method for delay differential equations. Commun Nonlinear Sci. Numer. Simulat., 2(4) (1997) 235236.

[9] M.A. Abdou and A.A. Soliman, New applications of variational iteration method. Phys. D, 211 (1-2) (2005) 1-8.

[10] S. Abbasbandy, Numerical solutions of nonlinear Klein-Gordon equation by variational iteration method. Internat. J. Numer. Meth. Engr., 70 (2007) 876-881.

[11] A.S. Arife and A. Yildirim, New modified variational iteration transform method (MVITM) for solving eighth-order boundary value problems in one step. World Appl. Sci. J., 13(10) (2011) 2186-2190.

[12] C. Rogers and W.F. Shadwick, Backlund Transformations, Academic Press, New York, 1982.

[13] T. L. Bock and M. D. Kruskal, A two-parameter Miura transformation of the Benjamin-One equation, Phys. Lett. A, 74 (1979) 173-176.

[14] M.L. Wang and X.Z. Li, Applications of F-expansion to periodic wave solutions for a new Hamiltonian amplitude equation, Chaos, Solitons and Fract., 24 (2005) 1257-1268.

[15] Z. Yan and H. Zhang, New explicit solitary wave solutions and periodic wave solutions for Whitham Broer-Kaup equation in shallow water, Phys. Lett. A, vol. 285 no.5-6 (2001) 355-362.

[16] M. Wang, Solitary wave solutions for variant Boussinesq equations, Phys. Lett. A, 199 (1995) 169-172.

[17] E.M.E. Zayed, H.A. Zedan and K.A. Gepreel, On the solitary wave solutions for nonlinear Hirota-Satsuma coupled KdV equations, Chaos, Solitons Fract., 22 (2004) 285-303.

[18] M. Inc and D.J. Evans, On traveling wave solutions of some nonlinear evolution equations, Int. J. Comput. Math., 81 (2004) 191-202.

[19] K, Khan and M.A. Akbar, Application of $\exp (-\varphi(\xi))$-expansion Method to Find the Exact Solutions of Modified BenjaminBona-Mahony Equation, World Appl. Sci. J, 24(10) (2013) 1373-1377.

[20] J.H. He and X.H. Wu, Exp-function method for nonlinear wave equations, Chaos, Solitons and Fractals, 30 (2006) $700-708$.

[21] J. Akter and M.A. Akbar, The $\exp (-\varphi(\xi))$ - expansion method for exact solutions to the nonlinear KdV Equation and the (2+1) dimensional Zakharov-Kuznetsov (ZK) equations, Elixir Appl. Math., 75 (2014) 27684-27692

[22] M.A. Abdou, The extended tanh-method and its applications for solving nonlinear physical models, Appl. Math. Comput., 190 (2007) 988-996.

[23] E.G. Fan, Extended tanh-function method and its applications to nonlinear equations, Phys. Lett. A, 277 (2000) 212-218. 
[24] W. Malfliet, Solitary wave solutions of nonlinear wave equations, Am. J. Phys., 60 (1992) 650- 654.

[25] M.J. Ablowitz and P.A. Clarkson, Solitons, Nonlinear Evolution Equations and Inverse Scattering Transform, Cambridge University Press, Cambridge, 1991,

[26] A.M. Wazwaz, A sine-cosine method for handing nonlinear wave equations. Math. Comput., 40 (2004) 499-508.

[27] S .Bibi, S.T.Mohyud-Din (2013) Traveling wave solutions of KdVs using sine-cosine method. J. Asso. Arab Univ. Basic and Appl. Sci., doi: org/10.1016/j.jaubas.2013.03.006 (in press).

[28] J.H. He and X.H. Wu, Exp-function method for nonlinear wave equations, Chaos, Solitons and Fractals, 30 (2006) $700-708$.

[29] M.A. Akbar , N.H.M. Ali, New Solitary and Periodic Solutions of Nonlinear Evolution Equation by Exp-function Method, World Appl. Sci. J., 17(12) (2012) 1603-1610.

[30] H. Naher, A.F. Abdullah and M.A.Akbar, New traveling wave solutions of the higher dimensional nonlinear partial differential equation by the Exp-function method, J. Appl. Math., Article ID 575387, (2012) 14 pages. doi: 10.1155/2012/575387.

[31] J.H. He, An elementary introduction to recently developed asymptotic methods and nano-mechanics in textile engineering, Int. J. Mod. Phys. B, 22(21) (2008) 3487-3578.

[32] R. Hirota, Exact envelope soliton solutions of a nonlinear wave equation, J. Math. Phys., 14 (1973) 805-810.

[33] R. Hirota and J. Satsuma, Soliton solution of a coupled KdV equation, Phys. Lett. A, 85 (1981) 407-408.

[34] Sirendaoreji, New exact travelling wave solutions for the Kawahara and modified Kawahara equations, Chaos Solitons Fract., 19 (2004) $147-150$

[35] J.H. He, Non-perturbative methods for strongly nonlinear problems, Dissertation. de-Verlag im Internet GmbH, Berlin, 2006.

[36] A. Biswas, G. Ebadi, M. Fessak, A. G. Johnpillai, S. Johnson, E. V. Krishnan and A. Yildirim, solutions of the perturbed KleinGordon equations, Iranian Journal of Science and Technology, Transaction A., 36 (2012) 431-452.

[37] A. Biswas, C. Zony and E. Zerrad, Soliton perturbation theory for the quadratic nonlinear Klein-Gordon equation. Appl. Math. Comput ., 203 (2008) 153-156.

[38] R. Sassaman and A. Biswas, Soliton perturbation theory for phi-four model and nonlinear Klein-Gordon equations, Communications in Nonlinear Science and Numerical Simulation, 14 (2009) 3239-3249.

[39] A. Biswas, A. Yildirim, T. Hayat, O. M. Aldossary and R. Sassaman, Soliton perturbation theory for the generalized Klein-Gordon equation with full nonlinearity, Proceedings of the Romanian Academy, Series A, 13 (2012) 32-41.

[40] M. Wang, X. Li and J. Zhang, The $\left(G^{\prime} / G\right)$-expansion method and travelling wave solutions of nonlinear evolution equations in mathematical physics, Phys. Lett. A, 372 (2008) 417-423.

[41] E.M.E. Zayed, Traveling wave solutions for higher dimensional nonlinear evolution equations using the $\left(G^{\prime} / G\right)$-expansion method, J. Appl. Math. Inform., 28 (2010) 383-395.

[42] M.A.Akbar, N.H.M. Ali and E.M.E. Zayed, Abundant exact traveling wave solutions of the generalized Bretherton equation via $\left(G^{\prime} / G\right)$-expansion method, Commun. Theor. Phys., 57 (2012) 173-178.

[43] M.A. Akbar, N.H.M. Ali and S.T. Mohyud-Din, Some new exact traveling wave solutions to the (3+1)-dimensional KadomtsevPetviashvili equation, World Appl. Sci. J., 16(11) (2012) 1551-1558.

[44] E.M.E. Zayed and K.A. Gepreel, The $\left(G^{\prime} / G\right)$-expansion method for finding the traveling wave solutions of nonlinear partial differential equations in mathematical physics, J. Math. Phys., 50 (2009) 013502-013514.

[45] M.A. Akbar and N.H.M. Ali, The alternative $\left(G^{\prime} / G\right)$-expansion method and its applications to nonlinear partial differential equations, Int. J. Phys. Sci., 6(35)(2011) 7910-7920.

[46] A.R. Shehata, The traveling wave solutions of the perturbed nonlinear Schrodinger equation and the cubic-quintic Ginzburg Landau equation using the modified $\left(G^{\prime} / G\right)$-expansion method, Appl. Math. Comput., 217 (2010) 1-10.

[47] M.A. Akbar, N.H.M. Ali and S.T. Mohyud-Din, The alternative $\left(G^{\prime} / G\right)$-expansion method with generalized Riccati equation: application to fifth order (1+1)-dimensional Caudrey-Dodd-Gibbon equation, Int. J. Phys. Sci., 7(5) (2012) 743-752.

[48] O. F. Gozukizil and T. Aydemir, Exact traveling wave solutions for some nonlinear parrrtial differential equations by using the $\left(G^{\prime} / G\right)$-expansion method.

[49] M. Alquran and A. Qawasmeh, Soliton sulutions of shallow water wave equations by means of $\left(G^{\prime} / G\right)$-expansion method, J.Appl. Ana.Comput., 4 (2014) 221-229 